\title{
Neues beim Pseudotumor cerebri (Idiopathische intrakranielle Hypertension)
}

\author{
Update on Pseudotumor cerebri (Idiopathic Intracranial \\ Hypertension)
}

Autoren

Jan Hoffmann, Arne May

Institut

Institut für Systemische Neurowissenschaften, Universitätsklinikum Hamburg-Eppendorf

Schlüsselwörter

Pseudotumor cerebri, Idiopathische intrakranielle Hypertension, Kopfschmerz

Key words

pseudotumor cerebri, idiopathic intracranial hypertension, headache

Bibliografie

DOI https://doi.org/10.1055/s-0043-113037 |

Akt Neurol 2017; 44: 466-475

(c) Georg Thieme Verlag KG Stuttgart · New York ISSN 0302-4350

\section{Korrespondenzadresse}

Priv.-Doz. Dr. med. Jan Hoffmann, Institut für Systemische Neurowissenschaften, Universitätsklinikum Hamburg-

Eppendorf, Martinistraße 52, 20246 Hamburg

j-r.hoffmann@uke.de

\section{ZUSAMMENFASSUNG}

Die idiopathische intrakranielle Hypertension (IIH) ist charakterisiert durch eine Liquordruckerhöhung unklarer Ätiologie. Klinisch äußert sich diese in Form von progredienten Sehstörungen, in der Regel als Folge eines Papillenödems, Kopfschmerzen und nicht selten Hirnnervenausfällen. In den letzten Jahren haben klinische Studien zunehmend zeigen können, dass das Syndrom häufig auch mit weiteren Symptomen wie Riechstörungen, kognitiven Defiziten und einem pulsierenden Tinnitus einhergehen kann.

Pathophysiologisch liegt der Erkrankung vermutlich eine venöse Abflussstörung zugrunde, die zu einer verminderten Liquorresorption und einem Anstieg des Liquordrucks führt, wobei unklar ist, ob die Abflussstörung auf einer übergewichtsbedingten Zunahme des intrathorakalen Drucks, dem Vorliegen von Stenosen im Sinus transversus oder beidem beruht. Seit einigen Jahren werden auch hormonelle Faktoren diskutiert, wobei deren Auswirkungen bislang weitgehend unklar sind.

Die Behandlung der IIH beruht vorwiegend auf einer effizienten Gewichtsreduktion sowie einer medikamentösen Behandlung mit Karboanhydrasehemmern, die sowohl die Liquorproduktion als auch das Körpergewicht reduzieren. Für den Karboanhydrasehemmer Azetazolamid liegen nun die Ergebnisse der ersten randomisierten, placebo-kontrollierten Studie einschließlich einer 12-monatigen Follow-up Phase vor, die zeigen, dass sich unter der Therapie sowohl das Papillenödem und die Sehstörungen als auch die Kopfschmerzen signifikant bessern. Für die Karboanhydrasehemmer Topiramat und Furosemid gibt es weiterhin nur offene, jedoch keine doppelblinden, placebo-kontrollierten Studien zur Wirksamkeit bei der IIH. Neben der Behandlung mit Karboanhydrasehemmern mehren sich inzwischen die Hinweise, dass das Somatostatinanalogon Octreotid wirksam in der Behandlung der IIH sein könnte, wobei für diese Substanz bislang keine randomisierten, doppelblinden, placebo-kontrollierten Studien vorliegen.

\section{ABSTRACT}

Idiopathic intracranial hypertension is characterized by an increase of intracranial pressure of unknown etiology. The clinical presentation is dominated by progressive visual disturbances, which are commonly the result of a papilledema, headache and cranial nerve palsies. Clinical studies have revealed over the past years that the syndrome may also be associated with olfactory disturbances, cognitive deficits and a pulsatile tinnitus.

The underlying pathomechanism is probably based on a disturbance of venous outflow which causes a reduced absorption of cerebrospinal fluid that results in an increase of cerebrospinal fluid pressure. It remains unclear if the venous outflow disturbance results from overweight-induced increase of intrathoracic pressure, the presence of sinus vein stenoses or both conditions. Recently hormonal factors have also been discussed but the mechanisms behind a potential influence on intracranial pressure remain unclear.

Treatment of idiopathic intracranial hypertension relies mainly on an effective body weight reduction and a medication with carboanhydrase inhibitors which can reduce the production of cerebrospinal fluid and body weight. Results of the first randomized, placebo-controlled trial as well as a 
12-month follow-up show that acetazolamide effectively reduces papilledema, visual disturbances and headache in idiopathic intracranial hypertension. In contrast, the evidence for the carboanhydrase inhibitors topiramate and furosemide still relies on open-label studies as doubleblind, placebo-controlled trials for their efficacy in idiopathic intracranial hypertension do not exist. In addition to the treatment with carboanhydrase inhibitors, increasing evidence suggests that the somatostatinanalogon octreotide may be effective in the treatment of idiopathic intracranial hypertension but up to date no randomized, double-blind, placebo-controlled trial exists to confirm this observation.

\section{Einleitung}

Das klinische Syndrom eines chronisch erhöhten Liquordrucks unklarer Ätiologie wurde erstmalig 1904 von dem deutschen Neurologen Max Nonne unter dem Begriff Pseudotumor cerebri (PTC) definiert. Im Laufe der Zeit wurden jedoch aufgrund der zunehmenden und effektiveren diagnostischen Möglichkeiten zahlreiche pathologische Zustände identifiziert, die eine chronische Steigerung des Liquordrucks hervorrufen können, sodass eine Einteilung in eine primäre und eine sekundäre Form notwendig wurde.

Seit einigen Jahren wird nun der Begriff „Pseudotumor cerebri“ als Überbegriff einer chronischen Steigerung des Liquordrucks verwendet, ohne weiter auf eine zugrunde liegende Ursache einzugehen. Der Pseudotumor cerebri unterteilt sich wiederum in eine primäre Form, die idiopathische intrakranielle Hypertension (IIH), und diverse sekundäre Formen, die im Rahmen anderer Erkrankungen wie beispielsweise Sinus- oder Hirnvenenthrombosen oder auch hormoneller Alterationen auftreten können [1,2]. Die folgende Übersichtsarbeit wird sich auf die primäre Form des PTC beschränken.

Die IIH hat eine Prävalenz von 8,6 Erkrankungsfällen auf 100000 Einwohner [3,4], wobei davon auszugehen ist, dass diese Zahl deutlich höher liegt, einerseits aufgrund der Tatsache, dass die existierenden epidemiologischen Studien einige Jahrzehnte zurückliegen und zu der Zeit andere Diagnosekriterien zugrundgelegt wurden, andererseits, weil die Erkrankung in ihrem klinischen Bild anderen primären Kopfschmerzerkrankungen wie beispielsweise der Migräne oder dem Spannungskopfschmerz, insbesondere deren chronischen Verlaufsformen, sehr ähneln kann und somit unterdiagnostiziert sein könnte [5, 6 ]. Hinzu kommt, dass an der IIH überwiegend übergewichtige Personen erkranken [7,8], sodass vor dem Hintergrund einer seit Jahren weltweit steigenden Prävalenz der Übergewichtigkeit auch von einer Zunahme der Prävalenz der IIH auszugehen ist [9]. Bei Erwachsenen betrifft die IIH in ca. $90 \%$ der Fälle Frauen in gebährfähigem Alter [10], wobei der Grund für die Geschlechtsgebundenheit weitgehend unklar ist, zumal bei erkrankten Kindern, inbesondere jungen, präpubertären Kindern, keine geschlechtsspezifischen Unterschiede in der Prävalenz nachweisbar sind [11-13].

\section{Pathophysiologie}

Liquorproduktion und -resorption stehen in einem fein justierten Gleichgewicht. Die initiale Vorstellung, dass der IIH eine Liquorüberproduktion zugrunde liegt, ist heute weitgehend widerlegt, weswegen die Forschungstätigkeiten heute auf Liquorresorption und venösen Abfluss konzentriert werden.

Die Tatsachen, dass IIH-Patienten meist sehr übergewichtig sind, der Liquordruck deutlich mit dem BMI korreliert und eine Gewichtsreduktion eine der effektivsten Behandlungsmöglichkeiten darstellt, suggerieren, dass ein übergewichtsbedingt erhöhter intraabdominaler und intrathorakaler Druck zu einer Zunahme des zerebralen Venendrucks und einer Abnahme der Liquorresorption führt, die letzten Endes eine Zunahme des Liquordrucks bewirken. Wenngleich diese Hypothese auf den ersten Blick sehr plausibel erscheint, so wirft sie einige Fragen auf. Sie erklärt beispielsweise nicht, warum überwiegend Frauen betroffen sind, zumal bei Frauen im Gegensatz zu Männern die Fettanreicherung eher im Beckenbereich als abdominal erfolgt. Da darüber hinaus die Mehrzahl der übergewichtigen Frauen nicht unter einer IIH leiden und Kinder mit einer IIH im Gegensatz zu Erwachsenen in der Regel sogar gar nicht übergewichtig sind, kann das reine Übergewicht den der IIH zugrunde liegenden Pathomechanismus nicht hinreichend erklären [13].

Vor diesem Hintergrund ist in den vergangenen Jahren spekuliert worden, dass möglicherweise diverse hormonelle Faktoren das verbindende Element zwischen dem Übergewicht und der IIH darstellen könnten. Daher werden in diesem Zusammenhang insbesondere hormonelle Prozesse diskutiert, die sich im Fettgewebe abspielen $[14,15]$. Beispielsweise ist bei IIH-Patienten die Leptin-Konzentration im Serum im Vergleich zu adipösen Kontrollprobanden ohne IIH signifikant erhöht, sodass eine zentrale Leptin-Resistenz diskutiert wird $[16,17]$. Interessanterweise ist die Leptin-Konzentration bei Frauen höher als bei Männern, sodass dieser Mechanismus auch erklären könnte, weswegen insbesondere Frauen von der IIH betroffen sind. Darüber hinaus ist bekannt, dass Geschlechtshormone, insbesondere Androgene, bei IIH-Patienten auffällig verändert sind. Auch eine potenzielle Rolle von Glukokortikoiden, insbesondere Kortisol, wird diskutiert, da dessen Verfügbarkeit durch die $11 \beta$-hydroxysteroid-dehydrogenase Typ 1 (11 -HSD1) reguliert wird. Da die 11ß-HSD unter anderem im Fettgewebe besonders aktiv ist und eine vermehrte Aktivität zu einer vermehrten Produktion von Kortisol führt, das seinerseits über die Wirkung am Glukokortikoid- und Mineralokortikoidrezeptor zu einer vermehrten Liquorproduktion führen könnte [15], 
würde dieser Mechanismus nicht nur die Entstehung des Überdrucks, sondern auch die weibliche Präponderanz erklären. Die aktuelle Datenlage reicht jedoch keineswegs aus, um hieraus konkrete pathophysiologische Schlüsse zu ziehen.

Eine weitere Hypothese besagt, dass eine oder mehrere Stenosen im Sinus transversus (TSS) den venösen Abfluss behindern und analog zum oben beschriebenen Mechanismus zu einem Anstieg des Liquordrucks führen. Für diese Hypothese spricht die Tatsache, dass TSS in ca. $90 \%$ der erwachsenen IIHPatienten festgestellt werden können und eine endovaskuläre Behandlung der Stenosen im Falle einer bilateralen TSS auch den IIH lindert oder beseitigt [18-20]. Dagegen spricht, dass hämodynamische Modelle zeigen, dass nur bilaterale TSS in der Lage sind, ein ausreichendes Abflusshindernis darzustellen, um die Liquorresorption signifikant zu beeinträchtigen [21]. Darüber hinaus kann auch dieser Mechanismus nicht erklären, warum überwiegend Frauen von einer IIH betroffen sind. Im Übrigen ist bislang nicht eindeutig geklärt, ob die TSS primär oder sekundär, d.h. Folge einer druckbedingten Kompression sind, da die TSS bei der IIH meist sehr langstreckig sind und in einigen Fällen nach Druckentlastung remittieren. Im Falle einer sekundären Genese würden die TSS jedoch möglicherweise zu einem Teufelskreis führen: Der hohe Liquordruck könnte zur Entstehung von TSS führen, die ein Abflusshindernis hervorrufen oder verstärken und darüber eine weitere Abnahme der Liquorresorption und Steigerung des Liquordrucks bewirken könnten. Letzten Endes würde dies zu einer weiteren sekundären Zunahme der TSS führen, sodass insgesamt eine Aggravation des Krankheitsbildes eintreten könnte. Da dieser Teufelskreis durch eine endovaskuläre Behandlung durchbrochen werden könnte, kann die Wirksamkeit der endovaskulären Behandlung nicht dazu beitragen, primäre von sekundären TSS zu differenzieren.

\section{Klinisches Bild und Diagnostik}

Die Diagnosekriterien der IIH sind aufgrund der zunehmenden diagnostischen Möglichkeiten, insbesondere der bildgebenden Verfahren, mehrfach modifiziert worden. Die aktuellen Diagnosekritieren von Friedman et al. basieren auf einer modifizierten Version der Dandy-Kriterien [1]. Die Diagnosestellung erfordert gemäß der derzeitigen Diagnosekriterien das Vorliegen eines Papillenödems, einer mit Ausnahme von Hirnnervenausfällen (insbesondere Abduzensparese) unauffälligen klinisch-neurologischen Untersuchung, eines cMRT ohne strukturelle Läsion, Anhalt für Hydrozephalus oder meningeales Enhancement, eine physiologische Liquorzusammensetzung sowie einen erhöhten Liquoreröffnungsdruck von $\geq 25 \mathrm{~cm}$ CSF bei Erwachsenen und $\geq 28 \mathrm{~cm}$ CSF bei Kindern (bei nicht-sedierten und normalgewichtigen Kindern $\geq 25 \mathrm{~cm}$ CSF) [1]. Neu in der aktuellen Fassung der Diagnosekriterien ist vor allem die Tatsache, dass die Diagnose alternativ auch bei Fehlen eines Papillenödems gestellt werden darf, wenn anstelle dessen eine uni- oder bilaterale Abduzensparese vorliegt. Sollte auch eine Abduzensparese nicht vorliegen, kann zwar keine gesicherte, aber zumindest eine wahrscheinliche Diagnose gestellt werden, sofern im cMRT eine Empty Sella, eine Abflachung des posterioren Anteils des Augapfels, eine Erweiterung des Subarachnoidalraums um den N. opticus (mit oder ohne geschlängeltem Verlauf des Sehnerven) und eine Stenose des Sinus transversus nachgewiesen werden können [1].

Jenseits der Diagnosekriterien wird das klinische Bild jedoch in erster Linie durch das Vorliegen von Kopfschmerzen, Sehstörungen sowie einer Reihe weiterer Symptome wie Hirnnervenlähmungen, Riechstörungen, kognitiven Defiziten und Tinnitus geprägt.

\section{Kopfschmerz}

Bis zu $90 \%$ der IIH-Patienten leiden unter Kopfschmerzen, sodass dieses Symptom zunächst meist führend ist und den Hauptgrund für die erste ärztliche Konsultation darstellt [7, 22]. Der IIH-assoziierte Kopfschmerz ist in den Diagnosekriterien der internationalen Kopfschmerzgesellschaft (ICHD-3 beta) definiert [23]. Dabei ist für die Diagnosestellung von Bedeutung, dass zeitgleich ein erhöhter Liquoreröffnungsdruck von $>25 \mathrm{~cm}$ CSF vorliegt und der Kopfschmerz zwei der drei folgenden Kriterien erfüllt: Kopfschmerz entwickelt sich im zeitlichen Zusammenhang zur IIH oder führt zu deren Diagnose, Kopfschmerz bessert sich nach Reduktion des intrakraniellen Liquordrucks, Kopfschmerz aggraviert im zeitlichen Zusammenhang mit einer Zunahme des intrakraniellen Liquordrucks. Damit ist im Vergleich zu den vorangegangenen Kopfschmerzkriterien von 2004 (ICHD-2) in den aktuellen Kriterien neu, dass eine Linderung der Kopfschmerzen nach Druckentlastung nicht mehr zwingend erforderlich ist [23]. Diese Änderung war dringend erforderlich, da zahlreiche Patienten auch lange Zeit nach Druckentlastung und auch langfristiger Normalisierung des Liquordrucks noch unter den chronischen Kopfschmerzen leiden können [24].

Die klinische Darstellung der IIH-assoziierten Kopfschmerzen ist äußerst variabel. Dies ist der Grund, weshalb die klinischen Charakteristika des Kopfschmerzes an sich aus den Diagnosekriterien entfernt worden sind und nur noch in den Kommentaren kurze Erwähnung finden [23]. Der Kopfschmerz wird als meist bilateral und frontal-retroorbital betont beschrieben, hat in der Regel einen dumpf-drückenden Charakter und tritt nahezu täglich auf $[23,24]$. Häufig berichten Patienten auch von einer Aggravation bei Husten, Pressen, Niesen oder auch bei körperlicher Belastung [24]. Der Kopfschmerz kann jedoch auch einseitig auftreten, einen teilweise pulsierenden Charakter aufweisen und sogar von Übelkeit sowie Photo- und Phonophobie begleitet sein, sodass eine Unterscheidung zu anderen primären Kopfschmerzerkrankungen wie zum Beispiel der chronischen Migräne durchaus sehr schwierig sein kann [7, 24, 25].

\section{Sehstörungen}

IIH-Patienten leiden häufig unter Sehstörungen. Diese sind in erster Linie auf ein druckinduziertes Papillenödem zurückzuführen, das sich bei der überwiegenden Mehrzahl der Patienten nachweisen lässt und sowohl ein- als auch beidseitig lokalisiert sein kann [7, 26-28]. Da inzwischen zweifelsfrei geklärt ist, dass die IIH auch ohne begleitendes Papillenödem auftreten kann, ist sowohl in den neuen Diagnosekriterien der IIH [1] als auch in denen des IIH-assoziierten Kopfschmerzes (ICHD-3 be- 
ta) [23] das Papillenödem als zwingendes Diagnosekriterium entfernt worden.

Das durch die chronische Liquordruckerhöhung induzierte Papillenödem ist in aller Regel progredient und führt zu zahlreichen Sehstörungen. Zu den am häufigsten beschriebenen Sehstörungen zählen Obskurationen, die Erweiterung des blinden Flecks, ein inferiorer bogenförmiger Gesichtsfelddefekt, Photopsien und ein Verlust der Sehschärfe [7,27]. Die Sehstörungen sind, wie das ihnen zugrunde liegende Papillenödem, progredient, und können, wenn eine effektive druckreduzierende Behandlung ausbleibt, zu irreversiblen Defiziten bis hin zur völligen Blindheit führen. Pathophysiologisch führt die chronische Druckerhöhung im Liquorraum auch zu einem Druckanstieg im Bereich der Optikusscheiden und einer resultierenden Drucksteigerung im Gewebe des Sehnerven. Diese Drucksteigerung führt zu Abnahme des Druckgradienten zwischen intraokulärem Druck und Liquordruck, der physiologisch vom Auge nach zentral verläuft [29, 30]. Dadurch kommt es zu einer einer Abnahme des axoplasmatischen Transports, was zu einer axonalen Schwellung im intrabulbären Teil des Sehnerven führt [30]. Der retrobulbäre Teil, in dessen Verlauf die Nervenfasern myelinisiert sind, ist davon nicht betroffen. Sekundär zu dieser axonalen Schwellung entsteht prälaminär eine Kompression von kleinen, insbesondere venösen, Kapillaren. Diese Alterationen können auch andere Erscheinungen wie beispielsweise kleine Hämorrhagien im Bereich der Papille zur Folge haben. Letzten Endes führen die genannten Veränderungen zu einer Atrophie der Papille mit einem irreversiblen Funktionsverlust des N. opticus [30]. Mit bildgebenden Techniken lassen sich sowohl die makroskopischen als auch die mikrostrukturellen Veränderungen, die aus der Druckerhöhung im Bereich der Optikusscheiden resultieren, zuverlässig darstellen [31-33].

Eine Korrelation zwischen dem Ausprägungsgrad des Papillenödems und dem Kopfschmerz ist nicht nachweisbar [34]. Möglicherweise ist dies durch die physiologische Enge in der Verbindung zwischen intrakraniellem Subarachnoidalraum und den Optikusscheiden begründet, die auch dafür verantwortlich gemacht wird, dass eine zum Teil mehrtägige Latenz zwischen akuten intrakraniellen Drucksteigerungen und der Entstehung eines Papillenödems liegen kann [30]. Hinzu kommt, dass der Liquordruck physiologischen Schwankungen im Tagesverlauf unterliegt, welche aufgrund der genannten Enge keine kurzfristigen Auswirkungen auf den Sehnerven haben können. Selbst wenn dies der Fall wäre, würde dies eine gewisse Zeit in Anspruch nehmen, um den axonalen Transport zu beeinflussen und visuelle Konsequenzen zu bewirken. Allerdings gibt es signifikante Korrelationen zu langfristigeren Parametern. So korreliert beispielsweise die Schwere des Papillenödems sowie der Verlust an Sehschärfe mit der Inzidenz einer Therapierefraktärität [35]. Gleiches gilt für den Nachweis von Hämorrhagien im Bereich der Papille, die bereits bei geringfügigen visuellen Beeinträchtigungen vorliegen können [36].

Aufgrund der Häufigkeit und der potenziellen Irreversibilität der visuellen Defizite und der damit einhergehenden langfristigen gravierenden Einschränkungen der Lebensqualität sollte zur diagnostischen Aufarbeitung eines Verdachts auf eine IIH in jedem Fall eine ausführliche opthalmologische Abklärung mit Funduskopie und Perimetrie erfolgen. In den letzten Jahren ist in diesem Zusammenhang zunehmend diskutiert worden, welchen Stellenwert eine optische Kohärenztomografie (OCT) in der Diagnostik eines IIH haben könnte. Die OCT stellt ein nicht-invasives Verfahren dar, welches u.a. eine hochauflösende und objektive Messung der Dicke des peripapillären retinalen Nervengewebes (retinal nerve fiber layer, RFNL) ermöglicht. Damit erscheint eine Anwendung bei der IIH zunächst sehr attraktiv, insbesondere für Verlaufskontrollen, die im Einzelfall auch sinnvoll sein kann $[37,38]$. Anhand einer OCT-Messung kann jedoch keine Aussage über den Zustand des Gewebes an sich getroffen werden. Das bedeutet, dass beispielsweise eine initial gemessene Verdickung der RFNL bei Papillenödem sich im Verlauf einer Erkrankung normalisieren kann, jedoch unklar bleibt, ob diese Normalisierung auf einer Abnahme der axonalen Schwellung oder dem Auftreten einer Papillenatrophie beruht [39]. Damit kann die OCT zwar eine opthalmologische Untersuchung mit Funduskopie und Perimetrie unterstützen, jedoch keineswegs ersetzen.

Neben den genannten diagnostischen Verfahren gewinnt auch die transorbitale sonografische Messung des Durchmessers der Sehnervenscheiden an Bedeutung in der Diagnostik der intrakraniellen Hypertension, da dieses Verfahren eine weitere nicht-invasive und rasch durchführbare Methode zur Identifikation verbreiteter Sehnervenscheiden und damit zur Stützung der Diagnose bietet [40-43]. Dieses Verfahren setzt jedoch eine weitreichende Erfahrung des Untersuchers voraus, um zuverlässige Ergebnisse zu erhalten. Aufgrund der relativ niedrigen Spezifität des Verfahrens kann die transorbitale sonografische Untersuchung weder die funduskopische Untersuchung noch die anderen diagnostischen Untersuchungen (Bildgebung, Liquordruckmessung) ersetzen.

\section{Hirnnervenlähmungen}

Im Rahmen einer IIH werden Hirnnervenlähmungen relativ häufig beobachtet. In den allermeisten Fällen handelt es sich dabei um Lähmungen des N. abducens, vereinzelt treten jedoch auch Lähmungen des N. oculomotorius [44], N. trochlearis [45], N. trigeminalis [46] oder N. facialis [47] auf, wobei auch mehrere Hirnnervenparesen gleichzeitig auftreten können [48]. In aller Regel bilden sich nach effektiver Behandlung des erhöhten Liquordrucks die Hirnnervenparesen wieder zurück $[47,49]$, sodass insgesamt von einer guten Prognose in Bezug auf diese Begleiterscheinungen auszugehen ist.

Die Abduzensparese geht stets mit horizontalen Doppelbildern einher und kann, wie auch die anderen Hirnnervenausfälle, uni- oder bilateral auftreten. Bei Erwachsenen tritt im Rahmen einer IIH eine Abduzensparese in ca. 10-20\% der Fälle auf [50], bei Kindern könnte die Inzidenz jedoch deutlich höher liegen [51]. IIH-Patienten mit begleitender Abduzensparese tendieren häufiger zu einem höheren BMI sowie zu einem höheren Liquordruck im Vergleich zu Patienten ohne Parese [51]. Wie es zu dem Ausfall des N. abducens im Rahmen des IIH kommt, ist nicht eindeutig geklärt, vermutet wird jedoch, dass der Hirnstamm im Rahmen der chronischen Steigerung des intrakraniellen Drucks diskret nach unten verdrängt wird, wodurch es am Dorello-Kanal im Bereich der Abduzensbrücke zu 
einer Traktion kommt, die den Nerven schädigt [51]. Dieser Pathomechanismus könnte auch eine hinreichende Erklärung dafür liefern, weshalb die Abduzensparese bei der IIH nicht selten bilateral auftritt. Es ist davon auszugehen, dass ähnliche Mechanismen auch den anderen $\mathrm{IIH}$-assoziierten Hirnnervenausfällen zugrunde liegen.

\section{Riechstörungen}

Das Auftreten von Riechstörungen bei der IIH ist erst seit wenigen Jahren bekannt $[52,53]$. Etwa $80 \%$ der IIH-Patienten leiden unter einer Hyposmie, die jedoch in der Regel leicht bis mittelgradig ausgepägt ist und möglicherweise aus diesem Grund lange unbeachtet blieb. Kunte et al. haben in einer klinischen Studie mit $17 \mathrm{IIH}$-Patienten und 17 alters- und geschlechtsgematchten gesunden Probanden das Auftreten von Riechstörungen bei der IIH systematisch mit Hilfe sog. „sniffin sticks“ untersucht. Anhand dieser Untersuchung lassen sich die Riechschwelle (threshold) sowie die Geruchsdiskrimination (discrimination) und -erkennung (identification) erfassen und sowohl einzeln beurteilen als auch zu einem TDI-Score verrechnen. Die Studie konnte zeigen, dass Geruchsdiskrimination und -identifikation bei der Mehrzahl der Patienten signifikant beeinträchtigt sind. Die Autoren konnten darüber hinaus zeigen, dass Patienten, deren Diagnose innerhalb der letzten drei Monate gestellt wurde und solche, deren Erkrankung sich in den letzten drei Monaten erheblich klinisch verschlechtert hatte, betroffener waren. Bei dieser Subgruppe ließ sich auch eine Abnahme der Riechschwelle nachweisen [53].

Die zugrunde liegenden Mechanismen sind bislang nicht eindeutig geklärt. Allerdings ist bekannt, dass bei der IIH strukturelle Veränderungen im N. olfactorius auftreten [54]. In diesem Zusammenhang konnte gezeigt werden, dass IIH-Patienten, insbesondere diejenigen, die eine kurze Krankheitsgeschichte hatten, ein reduziertes Volumen des Bulbus olfactorius aufwiesen [54]. Dieses ist höchstwahrscheinlich das strukturelle Korrelat für das häufig nachweisbare funktionelle Defizit [55-57]. Ob jedoch das reduzierte Volumen des Bulbus olfactorius lediglich Folge einer direkten Druckeinwirkung ist, ist nicht eindeutig geklärt. Die Tatsache, dass eine Druckentlastung über eine Liquorpunktion schon in kurzer Zeit zu einer erheblichen Verbesserung der olfaktorischen Fähigkeiten führt, legt dies jedoch nahe [58].

\section{Tinnitus}

IIH-Patienten berichten häufig von einem uni- oder bilateralen pulsatilen Tinnitus [7]. Die zugrunde liegende Ursache dieser Ohrgeräusche ist bislang nicht eindeutig geklärt. Die derzeit gängigste Hypothese geht jedoch davon aus, dass der pulsatile Tinnitus durch eine oder mehrere TSS entstehen könnte. Dabei wird postuliert, dass im Bereich der Stenose hörbare Turbulenzen im Blutfluss entstehen. Gestützt wird diese Hypothese durch die Tatsache, dass die invasive Beseitigung der Stenose anhand eines Stents häufig auch den Tinnitus beseitigt. Boddu et al. zeigten in einer prospektiven Studie mit $29 \mathrm{IIH}$-Patienten mit TSS und pulsatilem Tinnitus, die mit einem Stent im Sinus transversus versorgt wurden, dass alle behandelten Patienten noch am Tag des Eingriffs ihren Tinnitus verloren. Während der zweijährigen Follow-up-Phase zeigte sich bei $10 \%$ der Patienten eine Restenosierung mit erneutem Auftreten des Tinnitus. Wenngleich diese Daten sehr für einen kausalen Zusammenhang sprechen, müssen diese Daten durch weitere Studien bestätigt werden, bevor ein eindeutiger pathophysiologischer Zusammenhang als gesichert gelten kann, zumal bislang weder klar ist, wieviele der IIH-Patienten mit Stenose einen pulsatilen Tinnitus aufweisen, noch bei wievielen IIH-Patienten mit Tinnitus eine TSS identifiziert werden kann.

\section{Kognitive Defizite}

Seit einigen Jahren mehren sich die Hinweise kognitiver Defizite bei der IIH. Zunächst stützte sich die Vermutung auf Fallberichte und kleine klinische Studien, in denen einzelne kognitive Domänen isoliert untersucht wurden [59-61]. Yri et al. zeigten nun in einer prospektiven Studie, dass Patienten mit einer IIH häufig unter einer globalen kognitiven Dysfunktion leiden. Dabei ließen sich die größten Defizite in der Verarbeitungsgeschwindigkeit und Reaktionszeit nachweisen [62]. Unklar ist bislang der genaue Mechanismus, der zu den identifizierten Defiziten führt. Denkbar wäre eine direkte Auswirkung der intrakraniellen Drucksteigerung, dagegen spricht jedoch, dass trotz Normalisierung des intrakraniellen Drucks durch eine adäquate Therapie die kognitiven Defizite, zumindest in der erfassten Follow-up-Zeit von drei Monaten, nicht abnehmen [62]. Somit scheint einiges dafür zu sprechen, dass den kognitiven Defiziten eher komplexe und nachhaltigere Ursachen zugrunde liegen als eine simple mechanische Kompression durch die intrakranielle Drucksteigerung. Da Patienten mit einer IIH im Rahmen ihrer Erkrankung nicht selten unter einer depressiven Verstimmung leiden und diese an sich zu einer Beeinträchtigung der kognitiven Leistungsfähigkeit führen kann, ist nicht auszuschließen, dass auch ein solcher Zusammenhang der beschriebenen Assoziation zugrunde liegt. Trotz der nun zur Verfügung stehenden Daten sind jedoch weitere prospektive Studien mit längeren Follow-up-Phasen dringend notwendig, da die kognitiven Defizite nicht nur signifikant die Lebensqualität einschränken, sondern auch essenziell für die gesellschaftliche Teilhabe und berufliche Reintegration sind. Vor diesem Hintergrund, insbesondere auch der bislang unklaren Reversibilität der Defizite, sollte eine diagnostische Abklärung einer IIH auch eine neuropsychologische Testung beinhalten, um kognitive Dysfunktionen früh erkennen zu können.

\section{Liquordiagnostik}

Bei der klinischen Aufarbeitung der IIH ist die Durchführung einer Lumbalpunktion zur Messung des Liquoreröffnungsdrucks obligat. Dabei sollte sowohl die Messung des Liquoreröffnungsdrucks als auch eine Liquoranalyse zum Ausschluss sekundärer Ursachen erfolgen. Die Druckmessung sollte in liegender Position und im unsedierten Zustand erfolgen, um die Messwerte nicht zu beeinflussen. Nach den derzeitigen Diagnosekriterien ist bei einem Liquoreröffnungsdruck von $\geq 25 \mathrm{~cm}$ CSF bei $\mathrm{Er}$ wachsenen und $\geq 28 \mathrm{~cm}$ CSF bei Kindern von einer pathologischen Druckerhöhung auszugehen [1]. Dabei sollte stets berücksichtigt werden, dass der Liquordruck deutlichen Schwankungen, auch im Tagesverlauf, unterliegen kann. Sollte daher 
der begründete Verdacht auf einen PTC bestehen, sollte, in Abhängigkeit der klinischen Beschwerden, unter Umständen eine zweite Lumbalpunktion erwogen werden [63]. In seltenen und begründeten Fällen könnte auch eine vorübergehende kontinuierliche Druckmessung erforderlich sein $[64,65]$. Die Liquorzusammensetzung sollte jedoch unauffällig sein [1].

\section{Magnetresonanztomografie (MRT)}

Die MRT hat in den letzten Jahren in der Diagnostik der IIH zunehmend an Bedeutung gewonnen und sollte fester Bestandteil der diagnostischen Abklärung sein. Dabei gilt es zum einen Begleiterkrankungen auszuschließen, die eine sekundäre Drucksteigerung hervorrufen könnten, zum anderen IIH-spezifische Veränderungen zu identifizieren. Zu den bedeutendsten IIH-assoziierten Veränderungen im zerebralen MRT gehören die Empty sella, eine Abflachung des posterioren Augapfels, eine Verbreiterung der Optikusscheiden mit oder ohne geschlängeltem Verlauf des N. opticus sowie das Vorliegen von uni- oder bilateral lokalisierten TSS [1,33,66-68]. Aufgrund der Tatsache, dass TSS in bis zu $90 \%$ der IIH-Patienten nachweisbar und möglicherweise von ursächlicher Bedeutung sind, sollte neben einer strukturellen MRT in jedem Fall auch eine MR-Venografie (MRV) erfolgen [19]. Neben der Suche nach einer TSS dient die MRV allerdings auch dem Ausschluss von Sinus- oder Hirnvenenthrombosen. Dies ist von besonderer Bedeutung, da diese auch als klinisch inapparente Mikrothrombosen auftreten können, die jedoch ein venöses Abflusshindernis darstellen könnten, das zur intrakraniellen Drucksteigerung führt und somit unter Umständen wiederum zu einer sekundären durch äußere Druckeinwirkung bedingten Zunahme der TSS führen könnte [20]. Ob die TSS im Regelfall Ursache oder Konsequenz der intrakraniellen Drucksteigerung ist, ist bislang nicht eindeutig geklärt.

Zusammenfassend ist jedoch festzuhalten, dass die in der MRT identifizierten Veränderungen aufgrund deren Sensitivität und Spezifität zum jetzigen Zeitpunkt die Diagnose einer IIH zwar wesentlich unterstützen können, jedoch keineswegs eine Lumbalpunktion mit Messung des Liquoreröffnungsdrucks ersetzen können.

\section{Therapie}

Die Hauptziele der Behandlung der IIH sind die Erhaltung des Sehvermögens sowie die Linderung der Kopfschmerzen. Dabei sollte die Therapie stets einem multimodalen Ansatz folgen, der in erster Linie aus einer effektiven Gewichtsreduktion besteht, die durch eine pharmakologische Behandlung mit Karboanhydrasehemmern ergänzt wird. Invasive Therapieverfahren wie die Fenestrierung der Optikusscheiden, endovaskuläre Therapieverfahren und Shuntanlagen sollten nur in therapierefraktären Fällen zum Einsatz kommen, insbesondere dann, wenn ein signifikantes Risiko eines Verlusts des Sehvermögens besteht.

\section{Gewichtsreduktion}

Aufgrund der hochsignifikanten Korrelation zwischen Übergewicht und einer chronischen intrakraniellen Drucksteigerung sollte die Gewichtsreduktion stets den Hauptbestandteil der Behandlung darstellen. Zahlreiche Studien haben inzwischen belegt, dass die Gewichtsreduktion zu einer signifikanten Abnahme des intrakraniellen Drucks, des Papillenödems sowie der assoziierten Sehstörungen und der Kopfschmerzen führt [69]. Dabei können bereits geringe Veränderungen wesentlichen Einfluss auf den Verlauf der Erkrankung bewirken. Interessanterweise gilt dies auch für Patienten, die initial normalgewichtig sind [8]. Dabei ist stets darauf zu achten, dass nach erfolgreicher Gewichtsabnahme keine erneute Zunahme eintritt, da diese wiederum mit einem erhöhten Wiederauftreten der IIH assoziiert ist [70]. Sollte sich eine nachhaltige Gewichtsreduktion bei deutlicher Adipositas nicht erzielen lassen, sollte eine operative Verkleinerung des Magens in Erwägung gezogen werden, wobei dieses Therapieverfahren aufgrund seiner Invasivität auf ansonsten therapierefraktäre Fälle begrenzt werden sollte [71].

\section{Lumbalpunktion}

Die Lumbalpunktion stellt einen elementaren Bestandteil der diagnostischen Aufarbeitung der IIH dar. In der Therapie der IIH ist die Liquorpunktion aufgrund ihrer Invasivität jedoch nur von nachrangiger Bedeutung. Sie kann jedoch in therapierefraktären Situationen bei drohendem Sehverlust als überbrückende Maßnahme vor Durchführung eines endovaskulären oder operativen Verfahrens erwogen werden.

Die durch die Liquorpunktion induzierte plötzliche Druckentlastung führt in aller Regel zu einer vorübergehenden Reduktion der Kopfschmerzen, wobei streng genommen diese Beobachtung durch keine prospektive klinische Studie belegt ist. Gleiches gilt für den Einfluss der akuten Druckentlastung auf die anderen IIH-assoziierten Symptome. Interessanterweise kann die Reduktion der Kopfschmerzintensität bei einigen $\mathrm{IH}$ Patienten einige Wochen anhalten, obwohl die abgelassene Liquormenge bereits nach wenigen Stunden nachgebildet ist [72]. Auch ein für wenige Tage persistierendes durch die Punktion bedingtes Liquorleck kann diese Beobachtung nicht hinreichend erklären. Denkbar ist jedoch, dass die Druckentlastung zu einer Wiederherstellung eines stabilen Gleichgewichts zwischen Liquorproduktion und -resorption führt, indem die Abnahme des Liquordrucks zu einer Reduktion von sekundär druckinduzierten TSS und somit zu einer Verbesserung des venösen Abflusses und der Liquorresorption führt [73-76]. Weitere Studien sind jedoch erforderlich, um diese Hypothese zu bestätigen.

\section{Medikamentöse Behandlung}

Die medikamentöse Behandlung der IIH basiert in erster Linie auf Karboanhydrasehemmern. Die Karboanhydrase ist im Plexus choroideus lokalisiert und reguliert die Produktion von $\mathrm{Hy}$ drogenkarbonat $\left(\mathrm{HCO}_{3}{ }^{-}\right)$und darüber die Liquorsekretion [77]. Die Hemmung dieses Enzyms bewirkt eine Reduktion der Li- 
quorproduktion [78] und somit eine Reduktion des Liquordrucks [79].

Azetazolamid ist in der Behandlung der IIH der am häufigsten eingesetzte Karboanhydrasehemmer und wird in der Regel in einem Dosisbereich von 500-2000 mg eingesetzt. Obwohl dieser bereits seit vielen Jahren zur Behandlung der IIH eingesetzt wird, basierte der Einsatz bislang auf den Ergebnissen offener oder unkontrollierter Studien ohne Placebogruppe [80, 81]. Erst vor wenigen Jahren wurde die erste randomisierte, placebo-kontrollierte Studie zur Wirksamkeit von Azetazolamid in der Behandlung der IIH durchgeführt. Die Studie zeigte, dass Azetazolamid, das im Rahmen der Studie bis zu einer täglichen Dosis von $4 \mathrm{~g}$ eingesetzt wurde, sowohl das Papillenödem und die visuellen Defizite als auch die Kopfschmerzen besserte [82]. Trotz relativ häufiger gastrointestinaler Nebenwirkungen führte die Behandlung zu einer signifikanten Besserung der Lebensqualität [83]. Diese klinische Besserung hielt auch in all den genannten Aspekten über einen Follow-up-Zeitraum von $12 \mathrm{Mo-}$ naten an [84]. Azetazolamid führte in der Studie zu einer signifikanten Gewichtsabnahme, die vermutlich auch wesentlich zur Wirksamkeit beigetragen hat $[82,85]$.

Im Falle einer fehlenden Wirksamkeit, Unverträglichkeit oder Kontraindikation von Azetazolamid kann alternativ Topiramat in einem Dosisbereich von 50-200 mg eingesetzt werden. Bislang existieren jedoch keine randomisierten, placebo-kontrollierten Studien zum Nachweis der Wirksamkeit. Lediglich einige offene Studien ohne Placebogruppe deuten auf eine Wirksamkeit bei der IIH hin $[80,86]$. Zu den häufigsten Nebenwirkungen zählen Parästhesien, Stimmungsschwankungen und kognitive Nebenwirkungen [87]. Es wird davon ausgegangen, dass auch bei Topiramat der wesentliche Wirkmechanismus auf einer Hemmung der Karboanhydrase beruht. Topiramat führt jedoch auch häufig zu einer signifikanten Gewichtsabnahme, sodass dieser Effekt zur Wirksamkeit nicht unerheblich beitragen könnte.

Furosemid stellt eine weitere medikamentöse Behandlungsoption dar. Auch Furosemid hemmt die Karboanhydrase, senkt darüber die Liquorproduktion und somit den Liquordruck [88]. Der für die Behandlung der IIH eingesetzte Dosisbereich liegt in der Regel zwischen 30 und $80 \mathrm{mg}$ am Tag. Randomisierte, placebo-kontrollierte Studien zur Wirksamkeit bei der Behandlung der IIH gibt es bislang jedoch nicht.

Neben den genannten Karboanhydrasehemmern gibt es derzeit im Zusammenhang mit der IIH Hinweise für eine Wirksamkeit von Octreotid, einem langwirksamen, synthetischen Somatostatin-Analogon, das darüber hinaus die Wirkung des growth hormone $(\mathrm{GH})$ und insulin-like growth factor 1 (IGF-1) hemmt. Die existierenden Daten weisen darauf hin, dass Octreotid sowohl das Papillenödem und die assoziierten Sehstörungen als auch die Kopfschmerzen erheblich bessern könnte [8992]. Damit geht die Wirksamkeit über die von Octreotid bekannte analgetische Wirkung weit hinaus [93]. Der genaue Wirkmechanismus bei der IIH ist unbekannt. Der Gedanke einer Octreotid-Therapie entstand jedoch aus der Beobachtung, dass eine Behandlung mit GH oder IGF-1 einen sekundären PTC bewirken kann $[90,94]$. Die in den zitierten drei Studien behandelten Patienten litten jedoch unter einem IIH, d.h. es lagen keine pathologischen Hormonveränderungen vor. Bei allen Studien handelt es sich jedoch um offene Studien oder Fallberichte, sodass weitere Studien zur Prüfung der Wirksamkeit unerlässlich sind.

\section{Endovaskuläre Behandlung}

Im Falle des Nachweises einer oder mehrerer Stenosen im Sinus transversus kann eine endovaskuläre Stentanlage eine wirksame Behandlungsoption darstellen $[18,20]$. Der detaillierte Wirkmechanismus ist bislang nicht eindeutig geklärt, es wird jedoch davon ausgegangen, dass durch die Beseitigung der TSS der venöse Abfluss verbessert wird, was wiederum zu einer Zunahme der Liquorresorption und letzten Endes zu einer Abnahme des Liquordrucks führt $[95,96]$. Trotz der relativ guten Daten zur Wirksamkeit sollte die endovaskuläre Behandlung zunächst nur bei therapierefraktären Fällen erwogen werden, da zum einen nur begrenzte Langzeitdaten zu Wirksamkeit und Sicherheit vorliegen und zum anderen die Behandlung nicht selten von Komplikationen wie einer Stentverschiebung, In-StentThrombose oder einer Sinusperforation mit konsekutivem Subduralhämatom begleitet sein kann [97].

\section{Fenestrierung der Optikusscheiden}

Bei diesem invasiven Eingriff erfolgt eine Inzision in den Meningen, die den N. opticus umgeben. Der Wirkmechanismus ist bislang nicht abschließend geklärt. Es ist zwar davon auszugehen, dass durch die Inzision eine Abnahme des Liquordrucks um den N. opticus erfolgt, unklar ist jedoch, weswegen dieser Effekt persistiert, wenngleich die meningeale Läsion in kurzer Zeit abheilt und somit das Liquorleck beseitigt. Die Wirksamkeit zur Reduktion des Papillenödems und der damit verbundenen Sehstörungen wurde zwar bereits in zahlreichen Studien belegt, ausreichende Daten zur Langzeitwirksamkeit und -sicherheit gibt es jedoch bislang nicht $[98,99]$. Vor diesem Hintergrund sollte auch dieser Eingriff nur in besonderen therapierefraktären Fällen erwogen werden, in denen ein akutes Risiko eines irreversiblen Sehverlusts besteht [6].

\section{Shuntanlage}

Im Rahmen einer Shuntanlage wird eine Druckreduktion durch eine Umgehung des physiologischen Liquorabflusswegs erzielt. Dabei kann sowohl ein ventrikuloperitonealer als auch ein lumboperitonealer Shunt zum Einsatz kommen, wobei ersterer mit einem geringeren Risiko von Nebenwirkungen und notwendigen Shuntrevisionen verbunden ist und daher bevorzugt werden sollte [100]. Dennoch können bei dem Eingriff nicht ungefährliche Nebenwirkungen wie beispielsweise eine Shuntinfektion oder -dysfunktion auftreten. Zudem gibt es bislang nur wenige Daten zur Langzeitwirksamkeit und -sicherheit [101]. Diese deuten darauf hin, dass sich durch eine Shuntanlage zwar in den meisten Fällen ein positiver Einfluss auf das visuelle Defizit, nicht jedoch auf den Kopfschmerz erzielen lässt. Dazu kommt, dass aus unterschiedlichen Gründen bei mehr als der Hälfte der Patienten eine Shuntrevision erforderlich ist [101, 102]. Vor diesem Hintergrund sollte dieser Eingriff nur bei therapierefraktären Patienten mit signifikantem Risiko eines irreversiblen Sehverlusts in Erwägung gezogen werden. 


\section{Interessenkonflikt}

J. Hoffmann hat Honorare für Beratungstätigkeiten und/oder Tätigkeiten im Rahmen eines Advisory Boards für Allergan, Chordate Medical, Novartis und Autonomic Technologies Inc. (ATI) erhalten. Darüber hinaus hat er Honorare für Vortragstätigkeiten von Novartis, Allergan und Teva erhalten. Es besteht kein Interessenkonflikt zu den Inhalten dieses Manuskripts.

A. May hat in den vergangenen zwei Jahren erhalten und/oder erhält weiterhin Fördermittel von der Deutschen Forschungsgemeinschaft (DFG), dem Bundesministerium für Bildung und Forschung (BMBF) sowie der Europäischen Union. Darüber hinaus hat er einen „unrestricted grant von Chordate Medical. In den letzten 2 Jahren war bzw. ist er als Berater oder Vortragender für Allergan, Autonomic Tecnologies Inc. (ATI), ElectroCore, TEVA und Desitin tätig. Es besteht kein Interessenkonflikt zu den Inhalten dieses Manuskripts.

\section{Literatur}

[1] Friedman DI, Liu GT, Digre KB. Revised diagnostic criteria for the pseudotumor cerebri syndrome in adults and children. Neurology 2013; 81: 1159-1165

[2] Friedman DI. The Pseudotumor Cerebri Syndrome. Neurol Clin 2014; 32: $363-396$

[3] Durcan F], Corbett J], Wall M. The incidence of pseudotumor cerebri. Population studies in lowa and Louisiana. Arch Neurol 1988; 45: 875 877

[4] Radhakrishnan K, Ahlskog JE, Cross SA et al. Idiopathic intracranial hypertension (pseudotumor cerebri). Descriptive epidemiology in Rochester, Minn, 1976 to 1990. Arch Neurol 1993; 50: 78-80

[5] Hoffmann J, Goadsby PJ. Update on intracranial hypertension and hypotension. Curr Opin Neurol 2013; 26: 240-247

[6] Hoffmann J. Headache attributed to intracranial hypertension and hypotension. In: Mitsikostas DD, Pameleire K, Hrsg. Pharmacological management of headaches.1. Aufl. Berlin: Springer; 2015: 189-205

[7] Wall M, Kupersmith M], Kieburtz KD et al. The idiopathic intracranial hypertension treatment trial: clinical profile at baseline. JAMA Neurol 2014; 71: $693-701$

[8] Daniels AB, Liu GT, Volpe NJ et al. Profiles of obesity, weight gain, and quality of life in idiopathic intracranial hypertension (pseudotumor cerebri). Am J Ophthalmol 2007; 143: 635-641

[9] Almarzouqi S], Morgan ML, Lee AG. Idiopathic intracranial hypertension in the Middle East: A growing concern. Saudi J Ophthalmol 2015; 29: $26-31$

[10] Wall M. Idiopathic intracranial hypertension. Neurol Clin 2010; 28: $593-617$

[11] Ko MW, Liu GT. Pediatric idiopathic intracranial hypertension (pseudotumor cerebri). Horm Res Paediatr 2010; 74: 381-389

[12] Cinciripini GS, Donahue S, Borchert MS. Idiopathic intracranial hypertension in prepubertal pediatric patients: characteristics, treatment, and outcome. Am J Ophthalmol 1999; 127: 178-182

[13] Sheldon CA, Paley GL, Xiao R et al. Pediatric idiopathic intracranial hypertension: age, gender, and anthropometric features at diagnosis in a large, retrospective, multisite cohort. Ophthalmology 2016; 123 : $2424-2431$

[14] Mendelson CR, Simpson ER. Regulation of estrogen biosynthesis by human adipose cells in vitro. Mol Cell Endocrinol 1987; 52: 169-176

[15] Markey KA, Uldall M, Botfield H et al. Idiopathic intracranial hypertension, hormones, and 11 beta-hydroxysteroid dehydrogenases. J Pain Res 2016; 9: 223-232
[16] Ball AK, Sinclair A], Curnow S] et al. Elevated cerebrospinal fluid (CSF) leptin in idiopathic intracranial hypertension $(\mathrm{IIH})$ : evidence for hypothalamic leptin resistance? Clin Endocrinol (Oxf) 2009; 70: 863 869

[17] Lampl Y, Eshel Y, Kessler A et al. Serum leptin level in women with idiopathic intracranial hypertension. J Neurol Neurosurg Psychiatry 2002; 72: $642-643$

[18] Dinkin MJ, Patsalides A. Venous sinus stenting in idiopathic intracranial hypertension: results of a prospective trial. J Neuroophthalmol 2017; 37: 113-121

[19] Farb RI, Vanek I, Scott JN et al. Idiopathic intracranial hypertension: the prevalence and morphology of sinovenous stenosis. Neurology 2003; 60: $1418-1424$

[20] Ahmed RM, Wilkinson M, Parker GD et al. Transverse sinus stenting for idiopathic intracranial hypertension: a review of 52 patients and of model predictions. AJNR Am J Neuroradiol 2011; 32: 1408-1414

[21] Bedford THB. The effect of increased intracranial venous pressure on the pressure of the cerebrospinal fluid. Brain 1935; 58: 427-447

[22] Giuseffi V, Wall M, Siegel PZ et al. Symptoms and disease associations in idiopathic intracranial hypertension (pseudotumor cerebri): A case control study. Neurology 1991; 41: 239

[23] Headache Classification Committee of the International Headache Society. The International Classification of Headache Disorders, 3rd edition (beta version). Cephalalgia 2013; 33: 629-808

[24] Yri HM, Rönnbäck C, Wegener M et al. The course of headache in idiopathic intracranial hypertension: a 12-month prospective followup study. Eur J Neurol 2014; 21: 1458-1464

[25] Wall M. The headache profile of idiopathic intracranial hypertension. Cephalalgia 1990; 10: 331-335

[26] Wall M, White WN2nd. Asymmetric papilledema in idiopathic intracranial hypertension: prospective interocular comparison of sensory visual function. Invest Ophthalmol Vis Sci 1998; 39: 134-142

[27] Keltner JL, Johnson CA, Cello KE et al. Baseline visual field findings in the Idiopathic Intracranial Hypertension Treatment Trial (IIHTT). Invest Ophthalmol Vis Sci 2014; 55: 3200 - 3207

[28] Bidot S, Bruce BB, Saindane AM et al. Asymmetric papilledema in idiopathic intracranial hypertension. J Neuroophthalmol 2015; 35: $31-36$

[29] Jonas JB, Ritch R, Panda-Jonas S. Chapter 2 - Cerebrospinal fluid pressure in the pathogenesis of glaucoma. In: Giacinto B, Carlo N, Hrsg. New Trends in Basic and Clinical Research of Glaucoma: A Neurodegenerative Disease of the Visual System Part B (Progress in Brain Research 221). Amsterdam: Elsevier; 2015: 33 - 47

[30] Hayreh SS. Pathogenesis of optic disc edema in raised intracranial pressure. Progr Retin Eye Res 2016; 50: 108 - 144

[31] Schmidt C, Wiener E, Lüdemann L et al. Does IIH alter brain microstructures? - A DTI-based approach. Headache 2017; 57: 746- 755

[32] Hoffmann J, Schmidt C, Kunte $\mathrm{H}$ et al. Volumetric assessment of optic nerve sheath and hypophysis in idiopathic intracranial hypertension. AJNR Am J Neuroradiol 2014; 35: 513-518

[33] Hoffmann J, Huppertz H], Schmidt C et al. Morphometric and volumetric MRI changes in idiopathic intracranial hypertension. Cephalalgia 2013; 33: $1075-1084$

[34] Digre KB, Nakamoto BK, Warner JE et al. A comparison of idiopathic intracranial hypertension with and without papilledema. Headache 2009; 49: 185-193

[35] Wall M, Falardeau J, Fletcher WA et al. Risk factors for poor visual outcome in patients with idiopathic intracranial hypertension. Neurology 2015; 85: 799-805

[36] Wall M, Thurtell M]. Optic disc haemorrhages at baseline as a risk factor for poor outcome in the Idiopathic Intracranial Hypertension Treatment Trial. Br J Ophthalmol 2017. DOI: 10.1136/bjophthalmol2016-309852 [Epub ahead of print] 
[37] Kaufhold F, Kadas EM, Schmidt C et al. Optic nerve head quantification in idiopathic intracranial hypertension by spectral domain OCT. PLoS One 2012; 7: e36965

[38] OCT Sub-Study Committee for the Nordic Idiopathic Intracranial Hypertension Study. Baseline OCT Measurements in the Idiopathic Intracranial Hypertension Treatment Trial, Part I: Quality Control, Comparisons, and VariabilityBaseline OCT Measurements in IIHTT, Part I. Invest Ophthalmol Vis Sci 2014; 55: 8180 - 8188

[39] Rebolleda G, Muñoz-Negrete FJ. Follow-up of mild papilledema in idiopathic intracranial hypertension with optical coherence tomography. Invest Ophthalmol Vis Sci 2009; 50: 5197-5200

[40] Lochner P, Brigo F, Zedde ML et al. Feasibility and usefulness of ultrasonography in idiopathic intracranial hypertension or secondary intracranial hypertension. BMC Neurology 2016; 16: 85

[41] Moretti R, Pizzi B, Cassini F et al. Reliability of optic nerve ultrasound for the evaluation of patients with spontaneous intracranial hemorrhage. Neurocrit Care 2009; 11: 406 - 410

[42] Geeraerts T, Merceron S, Benhamou D et al. Non-invasive assessment of intracranial pressure using ocular sonography in neurocritical care patients. Intensive Care Med 2008; 34: 2062 - 2067

[43] Bäuerle J, Nedelmann M. Sonographic assessment of the optic nerve sheath in idiopathic intracranial hypertension. J Neurol 2011; 258: 2014-2019

[44] McCammon A, Kaufman HH, DSears ES. Transient oculomotor paralysis in pseudotumor cerebri. Neurology 1981; 31: 182-184

[45] Lee AG. Fourth nerve palsy in pseudotumor cerebri. Strabismus 1995; 3: $57-59$

[46] Davenport RJ, Will RG, Galloway PJ. Isolated intracranial hypertension presenting with trigeminal neuropathy. J Neurol Neurosurg Psychiatry 1994; 57: 381

[47] Capobianco DJ, Brazis PW, Cheshire WP. Idiopathic intracranial hypertension and seventh nerve palsy. Headache 1997; 37: 286-288

[48] Soroken C, Lacroix L, Korff CM. Combined VIth and VIIth nerve palsy: Consider idiopathic intracranial hypertension! Eur | Paediatr Neurol 2016; 20: $336-338$

[49] Davie C, Kennedy P, Katifi HA. Seventh nerve palsy as a false localising sign. J Neurol Neurosurg Psychiatry 1992; 55: 510-511

[50] Wall M, George D. Idiopathic intracranial hypertension. A prospective study of 50 patients. Brain 1991; 114: 155-180

[51] Reid JE, Reem RE, Aylward SC et al. Sixth nerve palsy in paediatric intracranial hypertension. Neuro-Ophthalmology 2016; 40: 23-27

[52] Khoo KF, Kunte H. Olfactory dysfunction in patients with idiopathic intracranial hypertension. Neurology 2014; 82: 189

[53] Kunte H, Schmidt F, Kronenberg $G$ et al. Olfactory dysfunction in patients with idiopathic intracranial hypertension. Neurology 2013; 81: $379-382$

[54] Schmidt C, Wiener E, Hoffmann J et al. Structural olfactory nerve changes in patients suffering from idiopathic intracranial hypertension. PLoS One 2012; 7: e35221

[55] Goektas O, Fleiner F, Sedlmaier B et al. Correlation of olfactory dysfunction of different etiologies in MRI and comparison with subjective and objective olfactometry. Eur J Radiol 2009; 71: 469-473

[56] Hummel T, Urbig A, Huart C et al. Volume of olfactory bulb and depth of olfactory sulcus in 378 consecutive patients with olfactory loss. J Neurol 2015; 262: 1046 - 1051

[57] Buschhüter D, Smitka M, Puschmann S et al. Correlation between olfactory bulb volume and olfactory function. Neuroimage 2008; 42 : $498-502$

[58] Becker N, Kronenberg G, Harms L et al. Rapid improvement of olfaction after lumbar puncture in a patient with idiopathic intracranial hypertension. Headache 2016. DOI: 10.1111/head.12802 [Epub ahead of print]
[59] Kaplan CP, Miner ME, McGregor JM. Pseudotumour cerebri: risk for cognitive impairment? Brain Inj 1997; 11: 293 - 303

[60] Sorensen PS, Thomsen AM, Gjerris F. Persistent disturbances of cognitive functions in patients with pseudotumor cerebri. Acta Neurol Scand 1986; 73: 264-268

[61] Kharkar S, Hernandez R, Batra S et al. Cognitive impairment in patients with Pseudotumor Cerebri Syndrome. Behav Neurol 2011; 24: $143-148$

[62] Yri HM, Fagerlund B, Forchhammer HB et al. Cognitive function in idiopathic intracranial hypertension: a prospective case-control study. BMJ Open 2014; 4: e004376

[63] Bono F, Salvino D, Tallarico T et al. Abnormal pressure waves in headache sufferers with bilateral transverse sinus stenosis. Cephalalgia 2010; 30: 1419-1425

[64] Torbey MT, Geocadin RG, Razumovsky AY et al. Utility of CSF pressure monitoring to identify idiopathic intracranial hypertension without papilledema in patients with chronic daily headache. Cephalalgia 2004; 24: 495-502

[65] Toma AK, Tarnaris A, Kitchen ND et al. Continuous intracranial pressure monitoring in pseudotumour cerebri: Single centre experience. $\mathrm{Br}$ J Neurosurg 2010; 24: $584-588$

[66] Agid R, Farb RI, Willinsky RA et al. Idiopathic intracranial hypertension: the validity of cross-sectional neuroimaging signs. Neuroradiology 2006; 48: $521-527$

[67] Degnan AJ, Levy LM. Pseudotumor cerebri: brief review of clinical syndrome and imaging findings. AJNR Am J Neuroradiol 2011; 32: $1986-1993$

[68] Maralani PJ, Hassanlou M, Torres C et al. Accuracy of brain imaging in the diagnosis of idiopathic intracranial hypertension. Clin Radiol 2012; 67: 656-663

[69] Sinclair A], Burdon MA, Nightingale PG et al. Low energy diet and intracranial pressure in women with idiopathic intracranial hypertension: prospective cohort study. BMJ 2010; 341: c2701

[70] Ko MW, Chang SC, Ridha MA et al. Weight gain and recurrence in idiopathic intracranial hypertension: a case-control study. Neurology 2011; 76: $1564-1567$

[71] Manfield JH, Yu KK-H, Efthimiou E et al. Bariatric surgery or non-surgical weight loss for idiopathic intracranial hypertension? A systematic review and comparison of meta-analyses. Obesity Surgery 2017; 27: $513-521$

[72] Johnston I, Paterson A. Beningn intracranial hypertension: II. CSF pressure and and circulation. Brain 1974; 97: 301-312

[73] Biousse V, Bruce BB, Newman NJ. Update on the pathophysiology and management of idiopathic intracranial hypertension. J Neurol Neurosurg Psychiatry 2012; 83: 488 - 494

[74] Scoffings DJ, Pickard JD, Higgins JN. Resolution of transverse sinus stenoses immediately after CSF withdrawal in idiopathic intracranial hypertension. J Neurol Neurosurg Psychiatry 2007; 78: 911 - 912

[75] Bateman GA, Stevens SA, Stimpson J. A mathematical model of idiopathic intracranial hypertension incorporating increased arterial inflow and variable venous outflow collapsibility. J Neurosurg 2009; 110: $446-456$

[76] De Simone R, Marano E, Fiorillo C et al. Sudden re-opening of collapsed transverse sinuses and longstanding clinical remission after a single lumbar puncture in a case of idiopathic intracranial hypertension. Pathogenetic implications. Neurol Sci 2005; 25: 342 - 344

[77] Damkier HH, Brown PD, Praetorius J. Cerebrospinal fluid secretion by the choroid plexus. Physiol Rev 2013; 93: 1847-1892

[78] Rubin RC, Henderson ES, Ommaya AK et al. The production of cerebrospinal fluid in man and its modification by acetazolamide. J Neurosurg 1966; 25: $430-436$ 
[79] Gucer G, Viernstein L. Long-term intracranial pressure recording in the management of pseudotumor cerebri. J Neurosurg 1978; 49: $256-263$

[80] Celebisoy N, Gokcay F, Sirin H et al. Treatment of idiopathic intracranial hypertension: topiramate vs acetazolamide, an open-label study. Acta Neurol Scand 2007; 116: $322-327$

[81] Ball AK, Howman A, Wheatley $\mathrm{K}$ et al. A randomised controlled trial of treatment for idiopathic intracranial hypertension. J Neurol 2011; 258: $874-881$

[82] Wall M, McDermott MP, Kieburtz KD et al. Effect of acetazolamide on visual function in patients with idiopathic intracranial hypertension and mild visual loss: the idiopathic intracranial hypertension treatment trial. JAMA 2014; 311: 1641 - 1651

[83] Digre KB, Bruce BB, McDermott MP et al. Quality of life in idiopathic intracranial hypertension at diagnosis: IIH Treatment Trial results. Neurology 2015; 84: 2449-2456

[84] Wall M, Kupersmith M], Thurtell MJ et al. The longitudinal idiopathic intracranial hypertension trial: outcomes from months 6-12. Am J Ophthalmol 2017; 176: $102-107$

[85] Sinclair AJ, Woolley R, Mollan SP. Idiopathic intracranial hypertension. JAMA 2014; 312: $1059-1060$

[86] Shah VA, Fung S, Shahbaz R et al. Idiopathic intracranial hypertension. Ophthalmology 2007; 114: 617

[87] Loring DW, Williamson DJ, Meador KJ et al. Topiramate dose effects on cognition: a randomized double-blind study. Neurology 2011; 76: $131-137$

[88] Carta F, Supuran CT. Diuretics with carbonic anhydrase inhibitory action: a patent and literature review (2005 - 2013). Expert Opin Ther Pat 2013; 23: $681-691$

[89] Deftereos SN, Panagopoulos G, Georgonikou D et al. Treatment of idiopathic intracranial hypertension: Is there a place for octreotide? Cephalalgia 2011; 31: 1679-1680

[90] House PM, Stodieck SRG. Octreotide: The IIH therapy beyond weight loss, carbonic anhydrase inhibitors, lumbar punctures and surgical/ interventional treatments. Clin Neurol Neurosurg 2016; 150: 181 184
[91] Panagopoulos GN, Deftereos SN, Tagaris GA et al. Octreotide: a therapeutic option for idiopathic intracranial hypertension. Neurol Neurophysiol Neurosci 2007; 10: 1

[92] Antaraki A, Piadites G, Vergados J et al. Octreotide in benign intracranial hypertension. Lancet 1993; 342: 1170

[93] Williams G, Ball JA, Lawson RA et al. Analgesic effect of somatostatin analogue (octreotide) in headache associated with pituitary tumours. Br Med J (Clin Res Ed) 1987; 295: 247 - 248

[94] Malozowski S, Tanner LA, Wysowski D et al. Growth hormone, insulin-like growth factor I, and benign intracranial hypertension. N Engl J Med 1993; 329: 665-666

[95] Owler BK, Parker G, Halmagyi GM et al. Cranial venous outflow obstruction and pseudotumor cerebri syndrome. Adv Tech Stand Neurosurg 2005; 30: 107-174

[96] Owler BK, Parker G, Halmagyi GM et al. Pseudotumor cerebri syndrome: venous sinus obstruction and its treatment with stent placement. J Neurosurg 2003; 98: 1045-1055

[97] Lueck C, Mcllwaine G. Interventions for idiopathic intracranial hypertension. Cochrane Database Syst Rev 2005: CD003434

[98] Spoor TC, McHenry JG. Long-term effectiveness of optic nerve sheath decompression for pseudotumor cerebri. Arch Ophthalmol 1993; 111: $632-635$

[99] Kelman SE, Heaps R, Wolf A et al. Optic nerve decompression surgery improves visual function in patients with pseudotumor cerebri. Neurosurgery 1992; 30: $391-395$

[100] Menger RP, Connor DEJr, Thakur JD et al. A comparison of lumboperitoneal and ventriculoperitoneal shunting for idiopathic intracranial hypertension: an analysis of economic impact and complications using the Nationwide Inpatient Sample. Neurosurg Focus 2014; 37: E4

[101] Sinclair AJ, Kuruvath S, Sen D et al. Is cerebrospinal fluid shunting in idiopathic intracranial hypertension worthwhile? A 10-year review. Cephalalgia 2011; 31: $1627-1633$

[102] Batra R, Sinclair A. Idiopathic intracranial hypertension; research progress and emerging themes. J Neurol 2014; 261: 451-460 